\title{
Hiperplasia Adrenal Congênita: Dosagem da 17-Hidroxiprogesesterona Basal para Seleção e Casos para Realização do Teste de Estímulo com ACTH Sintético
}

\author{
Congenital Adrenal Hyperplasia: Measurement of Basal 17-Hydroxyprogesterone as a \\ Screening Test to Select Patients for the Synthetic ACTH Provocative Test \\ Lúcia Helena Coelho Nóbrega, Josivan Gomes de Lima, Maria Lúcia Coelho Nóbrega, \\ Ana Luiza de Souza Brito, Raphael Pinto de Mendonça
}

\section{RESUM0}

\begin{abstract}
Objetivos: avaliar se os níveis de 17-hidroxiprogesterona podem predizer o resultado do teste de estímulo como diagnóstico de hiperplasia adrenal congênita, forma tardia.

Métodos: foram incluídas no estudo e avaliadas retrospectivamente 122 pacientes com suspeita clínica de hiperplasia adrenal congênita forma tardia. Essa suspeita clínica incluía sinais e/ou sintomas de hiperandrogenismo (hirsutismo, acne, pele oleosa, irregularidade menstrual, etc.). Todas as pacientes foram submetidas ao teste de estímulo da adrenal com ACTH sintético 0,25 mg (Synacthen ${ }^{\circledR}$ ). Após repouso de 60 minutos as amostras foram colhidas nos tempos basale 60 minutos após a administração de $0,25 \mathrm{mg}$ de ACTH sintético para dosagem de 17hidroxiprogesterona, sendo mantido o acesso venoso com catéter heparinizado. Foi utilizado o método de radioimunoensaio para realizar as dosagens séricas da 17-hidroxiprogesterona. A sensibilidade e a especificidade da 17-hidroxiprogesterona basal como teste de rastreamento para hiperplasia adrenal congênita foram medidas, avaliando vários pontos de corte. Curvas ROC foram feitas para analisar a performance do teste, utilizando o software Medcalc ${ }^{\circledR}$.

Resultados: a análise por curva ROC mostrou um ponto de corte de $181 \mathrm{ng} / \mathrm{dl}$ acima do qual dever-se-ia realizar o teste de estímulo, bem próximo a $200 \mathrm{ng} / \mathrm{dl}$, mais comumente aceito pela literatura. Niveis séricos da 17-hidroxiprogesterona mais altos que $200 \mathrm{ng} / \mathrm{dl}$ têm valores preditivo positivo e negativo de $75 \%$ e $100 \%$ e acurácia de $98,4 \%$ como diagnóstico de hiperplasia adrenal não-clássica.

Conclusões: considerando os dados, sugerimos que pacientes com hiperandrogenismo clínico devam iniciar a investigação com 17-hidroxiprogesterona basal e, caso esta se mostre acima de $181 \mathrm{ng} / \mathrm{dl}$, sigam a investigação com o teste de estímulo com ACTH sintético.
\end{abstract}

PALAVRAS-CHAVE: Hiperplasia adrenal congênita. 17-hidroxiprogesterona. Diagnóstico.

\section{Introdução}

A hiperplasia adrenal congênita (HAC) é doença que se origina de mutações no gene codificador de enzimas que compõem a via de síntese dos glicocorticóides na adrenal, cujo defeito encontra-se no cromossomo 6, com transmissão

Centro de Endocrinologia de Natal - RN

Correspondência:

Lúcia Helena Coelho Nóbrega

Centro de Endocrinologia de Natal - RN

Av Afonso Pena, 757 - Tirol

59020-100 - Natal - RN

e-mail: josivan@usa.net autossômica recessiva ${ }^{1}$. A deficiência da 21 hidroxilase é responsável por 90-95\% dos casos de hiperplasia adrenal ${ }^{2}$.

A gravidade do quadro clínico é muito variável, dependendo do nível androgênico, da eficiência da conversão dos precursores a andrógenos mais potentes e da expressão do receptor androgênico ${ }^{3}$. A deficiência da 21-hidroxilase pode ser grave, apresentando-se sob a forma perdedora de sal por deficiência de mineralocorticóides. Por outro lado, pode se apresentar com quadro clínico de hiperandrogenismo, exclusivamente. Este diagnóstico muitas vezes não é estabelecido devido à semelhança de apresentação clínica com a 
síndrome dos ovários policísticos e outros distúrbios androgenéticos ou mesmo porque o quadro clínico pode ser discreto ${ }^{2}$.

A HAC por deficiência de 21-hidroxilase pode se apresentar sob a forma clínica de início tardio, que geralmente se manifesta no período peri ou pós-puberal. Primeiramente relatada em 19584, também conhecida como não clássica (HAC-NC), atenuada, leve ou adquirida, acomete 1 a $6 \%$ das mulheres caucasianas com hiperandrogenismo ${ }^{5}$. A maior parte dessas pacientes são heterozigotos compostos com pequenas ou grandes mutações nos dois alelos ${ }^{6}$.

Além de o quadro clínico na maior parte das vezes ser muito semelhante ao da síndrome dos ovários micropolicísticos ${ }^{1}$, os níveis plasmáticos de testosterona e sulfato de deidroepiandrosterona também são similares e não há hipocortisolismo evidente. Tem sido sugerido que valores séricos de 17-hidroxiprogesterona basal na fase folicular menores ou iguais a $200 \mathrm{ng} / \mathrm{dL}$ afastariam a possibilidade diagnóstica da forma não clássica de HAC, embora valores acima de $200 \mathrm{ng} / \mathrm{dL}$ confirmem o diagnóstico com teste de estímulo ou análise genética em apenas $20 \%$ dos pacientes ${ }^{6}$. O melhor método diagnóstico laboratorial, com melhor correlação com a avaliação genética, ainda é o teste de estímulo da 17-hidroxiprogesterona com ACTH sintético ${ }^{7}$. No entanto, trata-se de exame de maior custo, de modo que estudos têm sido realizados com o objetivo de estabelecer um ponto de corte a partir do qual se indique a sua realização de forma custo-efetiva na grande população de mulheres com hiperandrogenismo.

O objetivo deste estudo foi avaliar a acurácia e o valor preditivo negativo da 17-hidroxiprogesterona basal, na fase folicular, para o diagnóstico de exclusão da HAC-NC, avaliando o ponto de corte sugerido na literatura (200 ng/dL) e propondo um valor baseado em nossos resultados.

\section{Pacientes e Métodos}

Foram incluídas no estudo e avaliadas retrospectivamente 122 pacientes atendidas no laboratório do Centro de Endocrinologia de Natal com suspeita clínica de HAC da forma tardia. Essa suspeita clínica incluía sinais e/ou sintomas de hiperandrogenismo (hirsutismo, acne, pele oleosa, irregularidade menstrual, etc.). Todas as pacientes foram submetidas ao teste de estímulo da adrenal com ACTH sintético 0,25 mg (Synacthen ${ }^{\circledR}$ ).

Todos os testes foram realizados no mesmo período do dia, pela manhã, após jejum de 12 horas, estando as pacientes na fase folicular do ciclo menstrual ou em amenorréia. Após repouso de 60 minutos as amostras foram colhidas, nos tempos basal e 60 minutos após a administração de 0,25 mg de ACTH sintético, para dosagem de 17-hidroxiprogesterona, sendo mantido o acesso venoso com cateter heparinizado. Foi utilizado o radioimunoensaio para realizar as dosagens séricas da 17-hidroxiprogesterona.

As pacientes foram divididas em dois grupos, de acordo com o resultado da 17-hidroxiprogesterona basal: grupo negativo ( $\leq 200 \mathrm{ng} / \mathrm{dL})$ e grupo positivo ( $>200 \mathrm{ng} / \mathrm{dL}$ ).

Valores de 17-hidroxiprogesterona após estímulo maiores que $1200 \mathrm{ng} / \mathrm{dL}^{8}$ foram considerados como confirmatórios de $\mathrm{HAC}-\mathrm{NC}$, sendo esse dado levado em conta para os cálculos de sensibilidade, especificidade, valores preditivos e curva ROC.

Os dados são mostrados como média \pm desvio padrão ou como mediana (p25-p75) quando não paramétricos. O coeficiente de Pearson (r) foi medido para avaliar a correlação entre os níveis basais de 17-hidroxiprogesterona e os observados 60 minutos após o estímulo. A sensibilidade e a especificidade da 17-hidroxiprogesterona basal como teste de rastreamento para HAC foram medidas, avaliando vários pontos de corte. Curvas ROC foram feitas para analisar a performance do teste, utilizando o software Medcalc ${ }^{\circledR 9}$. A análise estatística avaliou a sensibilidade, especificidade, valores preditivos positivo e negativo do valor basal de 17-hidroxiprogesterona no valor de $200 \mathrm{ng} / \mathrm{dL}$ como diagnóstico de HAC-NC, utilizando intervalo de confiança de 95\% (IC 95\%). A acurácia e índice kappa do limite de $200 \mathrm{ng} / \mathrm{dL}$ também foram avaliados e este foi considerado como muito bom se acima de $0,8^{9}$. Análise de tabela de contingência, usando teste exato de Fisher, foi feita usando o GraphPad $^{10}$. Estabeleceu-se como significância estatística $\mathrm{p}<0,05$.

\section{Resultados}

O teste de estímulo com ACTH sintético (Synacthen ${ }^{\circledR}$ ) foi realizado em 122 pacientes na fase folicular ou em amenorréia por suspeita de HAC-NC. A média de idade foi $16 \pm 10$ anos. O valor médio dos níveis de 17-hidroxiprogesterona basal foi 53,0 ng/dL (10,0-1505,0 ng/dL) e 60 minutos após estímulo foi 204,0 ng/dL (48,0-8700,0 ng/dL). A dosagem da 17-hidroxiprogesterona basal apresentou correlação direta com a 17-hidroxiprogesterona 60 minutos após estímulo ( $\mathrm{r}=0,92$; IC 95\%, 0,89-0,94; $\mathrm{p}<0,0001)$, com grau de concordância muito bom (indice kappa, 0,85$)^{9}$.

Com a análise por curva ROC, o valor de 17hidroxiprogesterona basal com melhor previsão 
diagnóstica para HAC-NC foi $181 \mathrm{ng} / \mathrm{dL}$, apresentando sensibilidade de $100 \%$ e especificidade de 98,3\% (Figura 1). Levando em conta o ponto de corte de 17-hidroxiprogesterona basal de $200 \mathrm{ng} / \mathrm{dL}$ como diagnóstico, conforme sugerido na literatura previamente, não foi observado nenhum caso de HAC-NC por deficiência de 21-hidroxilase no grupo negativo $(\mathrm{n}=114)$. Das pacientes do grupo positivo pela dosagem de 17-hidroxiprogesterona (basal >200 ng/dL; $\mathrm{n}=8$ ), $75 \%$ (6 pacientes) mostraram niveis de 17-hidroxiprogesterona após estímulo superior a $1200 \mathrm{ng} /$ $\mathrm{dL}$, configurando o diagnóstico laboratorial de hiperplasia adrenal congênita $(\mathrm{p}<0,0001$, Tabela 1$)$.

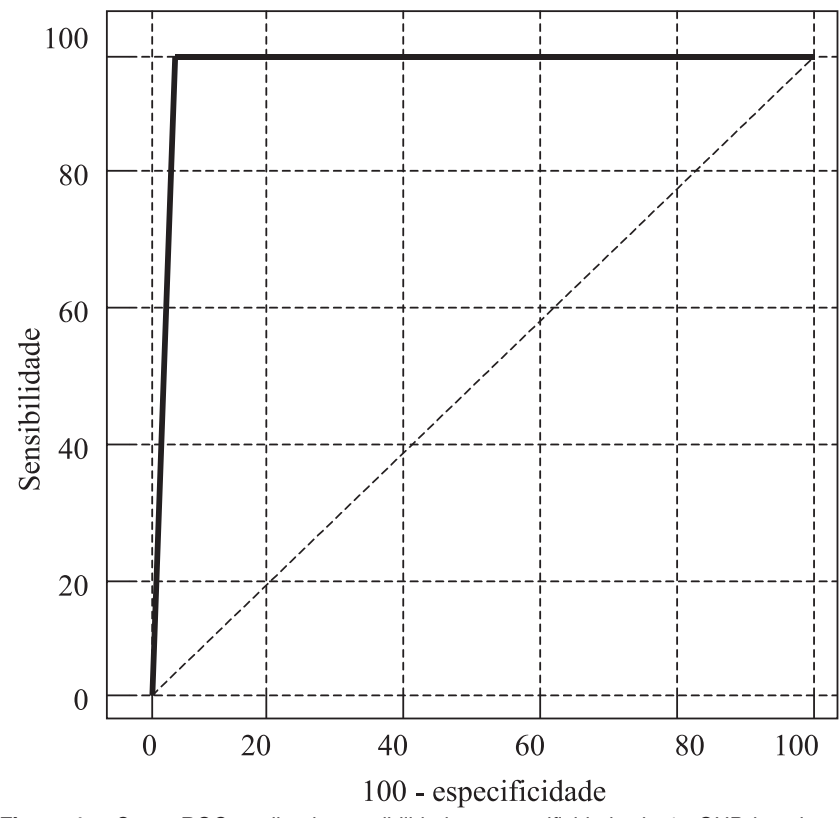

Figura 1 - Curva ROC avaliando sensibilidade e especificidade da 17-OHP basal.

Tabela 1 - Valores basais de 17-hidroxiprogesterona (17-OHP) e após estímulo.

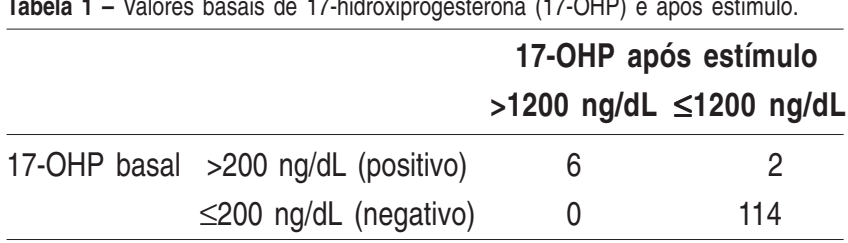

A média dos valores de 17-hidroxiprogesterona basal nas pacientes sem hiperplasia adrenal congênita foi 52,0 ng/dL (10,0-494,0 ng/dL), ao passo que nas pacientes com hiperplasia adrenal congênita foi 353,5 ng/dL (223,0-1505,0 ng/dL). Considerando o diagnóstico de HAC-NC, a dosagem da 17hidroxiprogesterona basal inferior a $200 \mathrm{ng} / \mathrm{dL}$ apresenta sensibilidade e valor preditivo negativo de $100 \%$, e acima de $200 \mathrm{ng} / \mathrm{dL}$, especificidade de $98,30 \%$ e valor preditivo positivo de $75,0 \%$. Desta forma o ponto de corte de $200 \mathrm{ng} / \mathrm{dL}$ para a 17-hidroxiprogesterona basal apresenta acurácia de 98,4\%.

\section{Discussão}

Pacientes com HAC-NC produzem quantidades normais de cortisol e aldosterona à custa de elevação leve a moderada de precursores androgênicos. Como a elevação desses precursores é discreta, a maioria dos casos não é detectada em investigação neonatal. Alguns casos, com o passar dos anos, tornam-se sintomáticos na infância com aceleração do crescimento, avanço da idade óssea e/ou pubarca precoce $^{11}$. Mais comumente, a HAC-NC apresentase com quadro semelhante à síndrome dos ovários policísticos, tendo hirsutismo como manifestação mais freqüente (60\% dos casos), além de amenorréia (54\%), acne (33\%) e infertilidade $(13 \%)^{12,13}$. Ademais, obesidade e irregularidade menstrual são achados associados em ambas as entidades clínicas ${ }^{1}$.

Além da semelhança da apresentação clínica, o quadro laboratorial também é bastante semelhante, sem diferença nas dosagens de testosterona, SDHEA ou androstenediona. Pacientes com sindrome dos ovários policísticos podem apresentar 17-hidroxiprogesterona até $20 \%$ acima do nível basal normal, já que este hormônio participa da esteroidogênese tanto adrenal como ovariana ${ }^{14}$.

Apesar de o teste de estímulo da 17-hidroxiprogesterona com ACTH sintético ser considerado o método laboratorial padrão-ouro para o diagnóstico de HAC-NC, esforços têm sido feitos para simplificar a investigação e selecionar pacientes de maior risco e que necessitem da realização do teste. Avaliando as nossas pacientes, com o uso de curva ROC, 17-hidroxiprogesterona basal de $181 \mathrm{ng} / \mathrm{dL}$ mostrou a melhor sensibilidade e especificidade como diagnóstico de HAC-NC (100 e 98,3\%, respectivamente).

A dosagem de 17-hidroxiprogesterona basal tem sido utilizada como investigação inicial, mas o ponto de corte a partir do qual deverá ser prosseguida a investigação ainda é controvertido. Alguns autores sugerem que o teste deveria ser realizado nas pacientes que apresentarem 17-hidroxiprogesterona basal maior que $200 \mathrm{ng} / \mathrm{dL}^{5}$. Outros consideram um valor máximo da 17-hidroxiprogesterona basal (acima de 500 ng/dL) que já seria diagnóstico de HAC, dispensando a realização do teste de estímulo e realizando-o apenas em valores intermediários (200-500 ng/dL) ${ }^{15}$. No entanto, o ponto de corte a partir do qual o teste seria dispensável ainda é bastante discutivel, uma vez que um diagnóstico falso-positivo implicaria tratamento errôneo com corticoterapia em pacientes que não se beneficiariam do seu uso, além da estigmatização da doença, o que implicaria inclusive planejamento genético pré-natal. Consideramos que todas as pacientes suspeitas deveriam ter o diagnóstico confirmado pelo teste de estímulo e, se necessário, por análise genética. 
Recentemente, alguns autores têm sugerido ponto de corte de $350 \mathrm{ng} / \mathrm{dL}$ para realização do teste de estímulo ${ }^{2}$. No entanto, considerando a análise da curva ROC em nossos dados, o ponto de melhor sensibilidade e especificidade seria de 181 ng/dL, aproximando-se, portanto, dos valores adotados na maioria dos trabalhos. Em nosso estudo, o ponto de corte de 17-hidroxiprogesterona basal sugerido na literatura ( $>200 \mathrm{ng} / \mathrm{dL}$ ), colhido na fase folicular, pela manhã, em jejum, demonstrou ser teste com acurácia de 98,4\%, muito boa para rastreamento diagnóstico de HAC-NC.

Não houve diferença no número de casos diagnosticados pelo teste de estímulo quando foram considerados os pontos de corte de $181 \mathrm{ng} / \mathrm{dL}$ ou de $200 \mathrm{ng} / \mathrm{dL}$. Entretanto, como a curva ROC detectou o valor $181 \mathrm{ng} / \mathrm{dL}$ como o de melhor sensibilidade e especificidade, de acordo com os nossos dados, sugerimos que todas as pacientes com suspeita clínica de HAC-NC devam realizar o teste basal e, caso este seja acima de $180 \mathrm{ng} / \mathrm{dL}$, realizar teste de estímulo com ACTH sintético.

\section{ABSTRACT}

Introduction: adrenal hyperplasia is a common genetic disorder and 95\% of the cases are due to a 21-hydroxylase deficiency. Clinical presentation varies from life-threatening salt-losing adrenal hyperplasia to simple androgenic states, which can be of late-onset and very similar to polycystic ovary syndrome. Diagnosis is usually made by synthetic ACTH provocative tests but efforts are being made to simplify this investigation.

Objective: to evaluate basal 17-hydroxyprogesterone as a predictor of the provocative test for the diagnosis of late-onset congenial adrenal hyperplasia. Methods: A total of 122 patients under clinical suspicion of diagnosis of late-onset congenial adrenal hyperplasia were included and retrospectively evaluated in the study. Such suspicion included signs and/or symptoms of hyperandrogenism (hirsutism, acne, oily skin, menstrual irregularity etc.). All the patients were submitted to the $0.25 \mathrm{mg}$ synthetic ACTH provocative test (Synacthen $($ )). After resting for 60 minutes, the samples were taken in the basal time and 60 minutes after the administration of $0.25 \mathrm{mg}$ synthetic ACTH, in order to assay 17-hydroxiprogesteron, the venous access being kept through a heparinized catheter. Radioimmuoessay was the method used to accomplish the assay of seric 17-hydroxiprogesteron. The sensitivity and specificity of the basal 17-hydroxiprogesteron were measured, assessing several cutoff points. ROC curves were made to analyze

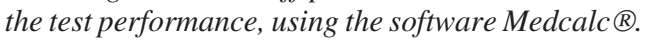

Results: ROC curve analysis showed that the best cutoff point was 181 $\mathrm{ng} / \mathrm{dl}$, which was very similar to the most common recommendation of $200 \mathrm{ng} / \mathrm{dl}$ of the literature. The cutoff point of $200 \mathrm{ng} / \mathrm{dl}$ shows positive and negative predictive values of 75 and $100 \%$, and accuracy of $98,4 \%$ as a diagnostic test for late-onset adrenal hyperplasia.

Conclusions: considering our data, we suggest that all hyperandrogenic patients should start the investigation with basal 17-hydroxyprogesteron and in case it is above $181 \mathrm{ng} / \mathrm{dl}$, then they should do the synthetic 17-hydroxyprogesteron provocative test.

\section{Agradecimentos}

Ao Prof. Dr. George Dantas de Azevedo pela revisão do manuscrito e sugestões.

\section{Referências}

1. Santos MC, Kater CE. Estudo de freqüência da hiperplasia adrenal congênita em centros de referência médica do Brasil. Arq Bras Endocrinol Metab 1998; 42:385-4.

2. Taboada GF, Teixeira RJ, Correa FHS, et al. Sensibilidade, especificidade e valor preditivo dos níveis basais da 17-hidroxiprogesterona no diagnóstico da forma não-clássica da hiperplasia adrenal congênita por deficiência da 21-hidroxilase. Arq Bras Endocrinol Metab 2003; 47:552-7.

3. White PC, Speiser PW. Congenital adrenal hyperplasia due to 21-hydroxylase deficiency. Endocr Rev 2000; 21:245-91.

4. Jayle MF, Weinmann SH, Baulieu EE, Vallin Y. Isolated post-puberal virilism in the absence of hydroxylation of steroids in $21 \mathrm{C}-21$ position. Acta Endocrinol (Copenh) 1958; 29:513-24.

5. Azziz R, Zacur HA. 21-Hydroxylase deficiency in female hyperandrogenism: screening and diagnosis. J Clin Endocrinol Metab 1989; 69:577-84.

6. Azziz R, Dewailly D, Owerbach D. Clinical review 56: nonclassic adrenal hyperplasia: current concepts. J Clin Endocrinol Metab 1994; 78:810-5.

7. Speiser PW, White PC. Congenital adrenal hyperplasia. N Engl J Med 2003; 349:776-88.

8. Marcondes JAM, Minanni SL, Sakamoto LC, Hayashida SY, Halbe HW, Wajchenberg BL. O espectro clinico e laboratorial da forma não clássica de hiperplasia adrenal congênita por deficiência da 21-hidroxilase. Arq Bras Endocrinol Metab 1995; 39:37-43.

9. Schoonjans F. Medcalc statistics for biomedical research: software manual [computer program]. Mariakerke: Medcalc Statistical Software; 2003.

10.Motulsky H. Analyzing data with GraphPad Prism. San Diego: GraphPad Software Inc, 1999.

11.Temeck JW, Pang SY, Nelson C, New MI. Genetic defects of steroidogenesis in premature pubarche. J Clin Endocrinol Metab 1987; 64:609-17.

12.Moran C, Azziz R, Carmina E, et al. 21-Hydroxylasedeficient nonclassic adrenal hyperplasia is a progressive disorder: a multicenter study. Am J Obstet Gynecol 2000; 183:1468-74.

13. Marynick SP, Chakmakjian ZH, McCaffree DL, Herndon $\mathrm{JH}$ Jr. Androgen excess in cystic acne. N Engl J Med 1983; 308:981-6.

14.Pinheiro SA, Clapauch R. Importância da dosagem da $17 \mathrm{OH}$-progesterona na síndrome dos ovários policísticos. Arq Bras Endocrinol Metab 2001; 45:361-8.

15.Dewailly D, Vantyghem MC, Lemaire C, Dufosse F, Racadot A, Fossati P. Screening heterozygotes for 21hydroxylase deficiency among hirsute women: lack of utility of the adrenocorticotropin hormone test. Fertil Steril 1988; 50:228-32.
KEYWORDS: Congenial adrenal hyperplasia. 17hydroxyprogesterone. Diagnosis.
Recebido em: 5/2/2004

Aceito com modificações em: 26/4/2004 